\title{
Improving the Supply System Gas Engine to Improve Energy Efficiency
}

\author{
Ramil Khakimov ${ }^{1 *}$, Otari Didmanidze ${ }^{2}$, and Ekaterina Parlyuk ${ }^{2}$ \\ ${ }^{1}$ Saint-Petersburg state agrarian university, 2 Peterburgskoe Highway, Saint-Petersburg, Pushkin, \\ 196601, Russia \\ ${ }^{2}$ Russian Timiryazev State Agrarian University,49 Timiryazevskaya str., Moscow, 127550, Russia
}

\begin{abstract}
The paper presents the main advantages and disadvantages of standard fuels in comparison with alternative fuels of methane series used in internal combustion engines. Particular attention in the article is given to the study of the heat transfer parameters of the calculated volume in the fuel tank filled with liquefied methane with thermal insulation, as well as methane outflow in special round tubes to transport gas in the liquid phase to the atomizer of the gas injector. Variants of numerical modeling of phase transitions of the heterogeneous system inside the tank are presented, as the calculated volume and flow of a two-phase flow of liquefied methane in tubes in the horizontal plane, taking into account the operating parameters of the elements of the fuel supply system.
\end{abstract}

\section{Introduction}

With the increasing number of road transport that is operated in large cities of Russia, the problem of saving of liquid petroleum fuel and reducing the toxicity of exhaust gases of internal combustion engines (CE) acquires in recent years increasingly important. With respect to technical, economic and environmental problems, scientists and engineers around the world solve this problem by improving the basic indicators of vehicle engines. This is achieved through energy efficiency systems and use of alternative fuels, including natural gas. Currently, natural gas (methane) is most widely used in compressed form. There is experience of use in internal combustion engines cryogenic methane [1,5].

By studying the power system of famous gas engines, we know as its advantages as disadvantages. Presenting gas engines belong to environmental class EURO-5, their advantages are obvious. Therefore, we analyze the deficiencies: - for the filling gas are used $80 \%$ of the high-pressure cylinders, mainly in compressed form - this complicates the layout and design of automotive vehicles and tractors; - the use of the gas - dosing device of electromagnetic type with a narrow range of adjustment of the characteristics it causes the incompleteness of combustion of methane-air mixture; - lack of an integrated electronic management system all systems gas engine - the absence of direct gas injection into the combustion chamber for improve combustion efficiency, energy consumption and

\footnotetext{
* Corresponding author: haki7@mail.ru
} 
environmental friendliness of the engine; - lack of ability to supply gas in the liquid phase to the spray caused by a number of daunting challenges for the implementation of this objective for the following reasons: the design of the atomizer, the solution of complex physical problems in terms of convective heat transfer at boundary conditions of low and high temperatures.

\section{Material and methods}

The above deficiencies constitute a complex problem in the implementation of individual organisational and technological stages in order to achieve integrated energy efficiency of gas engine power plants.

Consider the scheme (Figure 1) operation of gas installations with use of elements which allows to increase the efficiency of the engine.

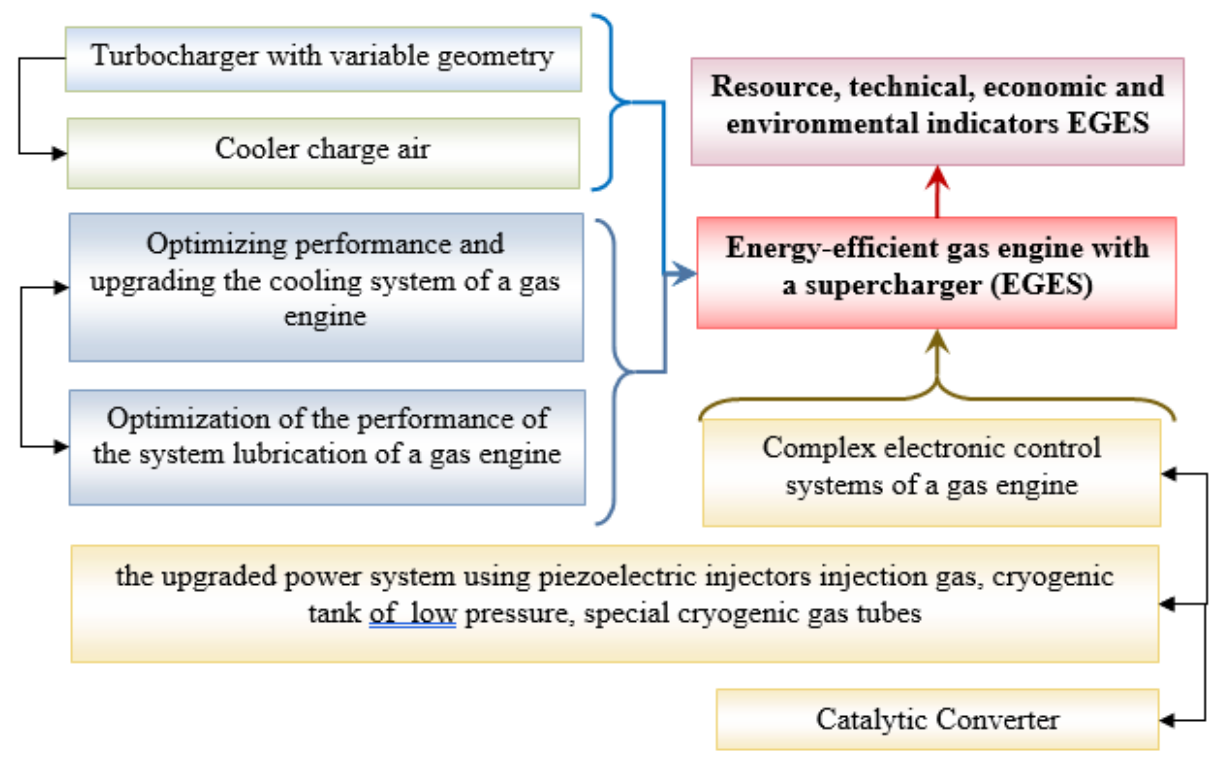

Fig. 1. The block diagram of the efficiency energy of the gas engine.

The solution to one of the above mentioned problems is the basis for scientific justification of the proposed project in the long term. Analysis of existing systems supply is doing same as in gas engines of vehicles - as well as for gas with external mixture formation or the distribution fuel injection $[2,15]$.

The most advanced and common systems of gas-cylinder equipment (GSE) 4th and 5th generation of the famous European producers basically sends the gas to the reducer in the compressed or liquefied state, but after the reducer gas is in the gaseous state to the ramp with fuel nozzle [3]. In practice, there are ways of feeding the gas directly into the combustion chamber but in gaseous form, mostly, the use of associated petroleum gases (propane and (or) butane), $[4,6]$. Flow directly into the combustion chamber of the methane currently does not exist, even in gaseous form. Theoretical and practical studies have shown that the use of methane as the primary fuel in full measure can be implemented only on the basis of the diesel engine in connection with the use of high strength and service life indicators The other important factor is the use of not the compressed and liquefied methane, and feeding methane in the liquid phase should occur before the reducer and the injector nozzle, and then directly into the combustion chamber $[9,11]$. 
This problem today is not solved from-for the arisen problems, the design of the injector and the physical behavior of liquid methane in the boundary conditions of simultaneous exposure to fields of low and high temperatures. This scheme of fuel in a gas engine is offered for the first time, so in practice no similar projects, but this problem is dealt with, for example, a Dutch company Vialle LPG released GSE 6th worship LPdi uses the technology of direct injection of compressed associated petroleum gas into the combustion chamber. And there is the project Direct4 Gas on the way to this goal, Robert Bosch $\mathrm{GmbH}$ is heading the consortium. The project partners are DaimlerAG and the Stuttgart research Institute of automotive technology and engines (FKFS). AG\&Co KG is an associated partner. The authors Seang-Wock Lee Prof. Kookmin University and Doo-Sung Baik Prof. Daejin University proposed constructive variant of the injector combined with spark plugs for compressed gas (associated petroleum gas) into the combustion chamber, this sample is presented in reference $[7,8]$.

All of the above companies are serious about to solve this problem, as it affects not only the result of energy efficiency, but also the superiority of the basic fuel economic and environmental performance among heat engines.

In this work, we consider the flow process of the liquid methane through the tube from the tank low pressure to the injector nozzle. For this we consider the diagram (Figure 2) the transportation of liquefied methane to the source of the spray gas in the combustion chamber.

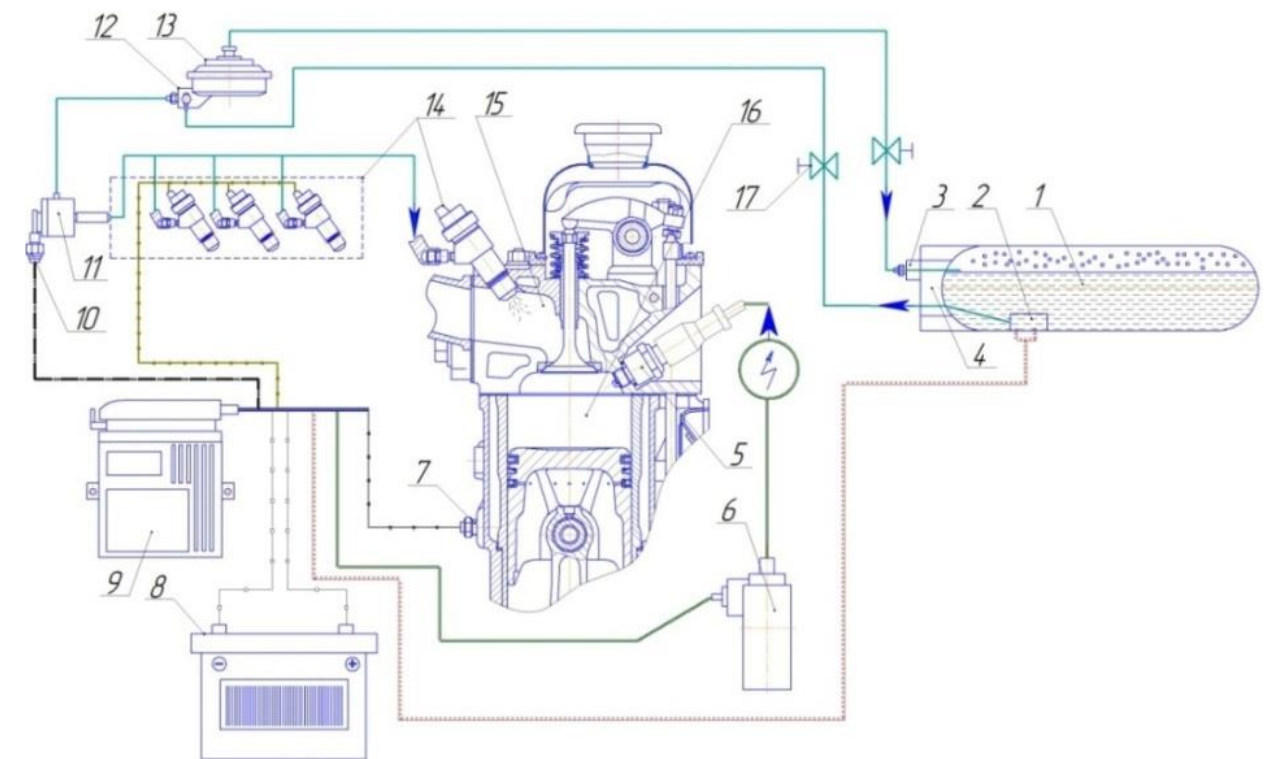

1 - gas (liquefied methane); 2 - pump; 3 - fuel consumer reverse action; 4 - the gas distributor; 5 ignition plug; 6 - ignition coil; 7 - temperature sensor of the cooling system; 8 - battery; 9 - electronic control unit; 10 - fuel temperature sensor; 11 - fuel pressure sensor; 12 - check valve main gas pipeline; 13 - gas reducer for collecting the gaseous phase; 14 - piezoelectric gas injector; 15 -intake path; 16 the combustion chamber; 17 - main safety valve.

Fig. 2. Diagram electronic control gas engine when operating on liquefied methane

From various literature sources $[12,26]$ it is known that methane in the liquid phase has a temperature equal to $-161^{\circ} \mathrm{C}$, this condition makes difficult the transportation of methane in liquefied form by special tubes to solve this problem it is necessary to conduct studies of the effect of heat transfer through the tube wall in a liquefied environment of methane at low temperatures. 


\section{Theory}

Consider the methodology and some results of calculating the storage of methane in a cryogenic tank, the cross section of the tank shown in figure 3.

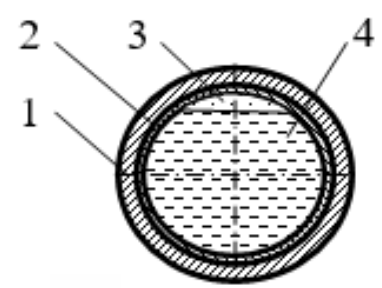

a)

1 - the first insulating layer; 2 - the second insulating layer; 3 - gaseous methane; 4 - liquefied methane; $I n$ - the upper area; $N$ - lower area; $L$ - left area, $P$ - right area

Fig. 3. a) is the cross section of the fuel tank, b) the volumetric mesh.

This mathematical model, which is described for the cryogenic hydrogen Jankov G. G., was adopted for the numerical simulation of cryogenic methane, and includes the presence of two phases: liquid $(k=1)$, gas $(k=2)$ and solid $(k=3)$ with a movable heterogeneous boundary "liquid-gas" $[13,16]$. The processes of heat and mass transfer inside the tank is described separately for the two phases of liquid and gas in Cartesian coordinates:

$$
\begin{gathered}
\frac{\partial \rho_{k}}{\partial \tau}+\nabla \cdot\left(\rho_{k} \vec{V}_{k}\right)=0, \\
\frac{\partial\left(\rho_{k} V_{k . x}\right)}{\partial \tau}+\nabla \cdot\left(\rho_{k} \vec{V}_{k} V_{k . x}-\rho_{k} v_{v . e f f} \nabla V_{k . x}\right)=-\frac{\partial \rho_{k}}{\partial x}+g_{x} \rho_{k}, \\
\frac{\partial\left(\rho_{k} V_{k . y}\right)}{\partial \tau}+\nabla \cdot\left(\rho_{k} \vec{V}_{k} V_{k . y}-\rho_{k} v_{v . e f f} \nabla V_{k . y}\right)=-\frac{\partial \rho_{k}}{\partial y}+g_{y} \rho_{k}, \\
\frac{\partial\left(\rho_{k} h_{k}\right)}{\partial \tau}+\nabla \cdot\left(\rho_{k} \vec{V}_{k} h_{k}-\lambda_{k . e f f} \nabla T_{k}\right)=\frac{\partial p_{k}}{\partial \tau}+Q_{r . k}, \\
\lambda_{k . e f f}=\lambda_{k}+\rho_{k} c_{p . k} \frac{v_{k . t}}{p_{\Gamma_{t}}}, \text { accept } \mathrm{k}=1,2,
\end{gathered}
$$

where $\rho_{k}$ - where is the density of the considered phase; $v_{k}$ - reduced mass-average velocity of the considered phase; $\lambda_{k, \text { eff }}-$ is the coefficient of effective thermal conductivity of the considered phase; $\vec{V}_{k}$ - vector of speed gas; $T_{k}$ - the temperature of this environment; $c_{p, k}-$ is the specific heat at constant pressure of the considered phase; $Q_{r, k}-$ is the heat flow; $h_{k}$ - length of the outlet pipe; $\tau$ - time cooling.

The temperature of the bounding walls of the tank was calculated according to the equation:

$$
\rho_{3} c_{3} \frac{\partial T_{3}}{\partial \tau}-\nabla \cdot\left(\lambda_{3} \nabla T_{3}\right)=Q_{V .3}+Q_{r .3}
$$


The main terms in the equations (4) and (5) are introduced for modeling heat transfer by radiation between wall and fluid, as well as to account for the heat generated by the wall from the environment.

Due to the small velocities of both phases in equations (4), you can omit the first term in right side describing the work of pressure forces. It is also assumed that the gas in a compressed form obeys the ideal gas law.

Given some assumptions, the equation of conservation of energy for phase $k$ looks follows:

$$
\rho_{k} \frac{\partial h_{k}}{\partial \tau}=\nabla \cdot\left(\frac{\lambda_{k . e f f}}{c_{p k}} \nabla h_{k}\right)+Q_{r . k} .
$$

Considering the physical properties of the test medium in the tank at the interface "liquid - gas" there are known physical phenomena such as surface tension, shear stress, etc., which can be neglected and the change in internal energy of phase boundaries, where phase adjustment $[14,22]$. On the wall of the tank to set the initial value of the velocity of gas and liquid which are reflected in detail in reference [16]. The solution of the problem in the design plan is rather complicated for two fundamental reasons.

The first complicating fact is that the position and shape of the interface "liquid-gas" is not known in advance, and are determined from the solution of a system which was formulated earlier in equations. The drain-free storage of the change in the position of the interfacial surface is negligible compared to the initial state, its form has a good approximation and can be flat. Therefore, the mathematical model and numerical method was designed for the General case (for example, the emptying of the tank under the action of a jet of the injected gas).

The second circumstance is that the interface "liquid-gas", "liquid - solid body" do not fit in any of the orthogonal coordinate systems. This feature necessitates the application of the method, the blocked cells [10], or the method of fractional cells for the first time, - this is method is proposes first $[17,21]$.

The investigated area of an arbitrary shape is divided into zones, each of which is occupied by only one phase. The zones are phase surfaces in two-dimensional case are described by the closed broken lines. The calculated volume through which the phase boundary, are fractional. The number of line segments in the phase interface, passing through the estimated fractional volume, can be arbitrary.

Integrating the equation of conservation of mass (1), bring him to the next expression

$$
\frac{\left(\rho_{p}-\rho_{p}^{0}\right)}{\Delta \tau} \Delta V_{p}+F_{e}+F_{w}+F_{n}+F_{s}+F_{f}=0,
$$

where $F_{f}=\vec{n}_{f} \cdot \rho_{f} \vec{V}_{f} \Delta S_{f}$ - mass flows across the faces of the estimated volume and micro-segments of the phase boundary, $\vec{n}_{f}$ - is the unit vector of external normal to the face of the estimated volume or phase boundary $(j=\{e, w, n, s, f\})$.

$$
a_{p} V_{x . p}=G_{p}^{x}-\int_{\Delta V p} \frac{\partial p}{\partial x} d V ; \quad a_{p} V_{y \cdot p}=G_{p}^{y}-\int_{\Delta V p} \frac{\partial p}{\partial y} d V ;
$$

To complete the system of equations (7), (8) it is necessary to calculate the pressures and mass flows through the edge of the calculation volume and microtrace phase boundaries. For this purpose the approach [18], adapted to the fractional case the calculated volumes. 
The pressure values at the boundaries of the calculation volume are calculated by the formulas:

$$
\begin{gathered}
p_{e}=p_{P e}+\frac{\left(x_{e}-x_{p}\right)}{\Delta x_{e}\left(p_{E_{\rho}}-p_{p_{\rho}}\right)}, \\
p_{P e}=p_{p}-\Delta p_{P e}=p_{p}-\left(\frac{\partial p}{\partial y}\right)_{p}\left(y_{p}-y_{P e}\right), \\
p_{E e}=p_{p}-\Delta p_{E e}=p_{E}-\left(\frac{\partial p}{\partial y}\right)_{E}\left(y_{E}-y_{E e}\right) .
\end{gathered}
$$

The calculations of the bulk environment in the fuel tank showed the theoretical validity of the mathematical model, numerical method the fractional calculation of volumes and implementing their software.

The materials that were used to develop fuel liquefied methane to the actuator (gas injector) is presented in $[19,23]$. Next, we consider the model of transportation of LNG by special pipes to the actuator. Typically, the objective in solving the problem is as follows to create special conditions in the tube for liquid methane to the environment was singlephase without the formation of gaps in boundary conditions properties gas. In practice, the expiration of liquefied natural gas will always be accompanied by the formation of two-phase flow in the tubes of the methane due to chemical-physical properties, hence consider first the model steady-state two-phase flow of liquid methane [24,25].

The one-dimensional equation of momentum and of continuity for two-phase flow of liquefied methane in the channel with round cross section can be represented as follows:

$$
\begin{gathered}
\frac{d p}{\partial z}+4 \frac{\tau}{D}+g \rho_{2 f} \cos \theta+\frac{d}{d z}\left\{\rho_{L} \alpha u_{G}^{2}+\rho_{L}(1-\alpha) u_{G}^{2}\right\}=0, \\
\left(\frac{d}{\partial x}\right)\left\{\rho_{G} \alpha u_{G}+\rho_{L}(1-\alpha) u_{L}\right\}=0,
\end{gathered}
$$

$D$ - where is the diameter of the tube; $\alpha$ - the heat transfer coefficient.

where the density of two-phase mixture is given by:

$$
\rho_{2 f}=\alpha \rho_{G}+(1-\alpha) \rho_{L}=0
$$

The second equation is a formulation of the law of conservation of mass mixture of liquid and gas phases. The speed of the two phases of gas and liquid is connected with the total mass flow equations:

$$
u_{G}=\left(\frac{x}{\alpha \rho_{G}}\right) G ; \quad u_{L}=\frac{(1-x) G}{(1-\alpha) \rho_{L}} .
$$

Taking into account expressions (12) loss of total pressure can be written in differential form:

$$
-\frac{d p}{d x}=\frac{\frac{4 \tau}{D}+g \rho_{2 f} \cos \theta}{1-G^{2}(-d v / d p)} .
$$


With regard to the pressure losses in two-phase flow, the local gas content is calculated from the energy equation, based on the proposal of thermodynamic equilibrium, then we determine the change of momentum at the loss of total pressure $G^{2} \Delta v$ :

$$
x=\frac{h_{O}-h_{\text {sat. }}+q-E_{k}}{h_{f}} .
$$

This assumption depends on the geometry of the flow and parameters of fuel injection systems. Kinetic energy is usually negligible, but can be used for the calculation. In addition to determining the gaseous content in the tube, it is also necessary to use an independent value for the local volume fraction of gas $\alpha$, and the specific volume of the mixture $v$.

\section{Results}

According to the research results it can be noted that the methane in a liquefied state has a rather complex physical state when transmitting simultaneously for low and high temperatures. The positive side of the issue is that there is every reason for the realization of this goal with proper selection of materials for further development and experimental samples of the constituent elements of the power supply system of a gas engine such as a cryogenic tank, pipes and gas nozzles [20,24].

The basis of the numerical simulation of a cryogenic fuel system of automotive tractor technology was made some conditions under which the following were taken into account: 1) the level of liquefied natural gas in a cryogenic tank at 50\% and $100 \%$ refueling; 2) the ambient temperature at which studies were carried out, mainly transitional and warm periods.

With long-term thermal effects (solar or (and) thermal inflow from the internal combustion engine) on the tank walls in experiment No. 1, 2, 3 (see Fig. 4), the correspondence between the experimental and calculated indicators in the mode of drainagefree liquefied storage is well observed. methane.

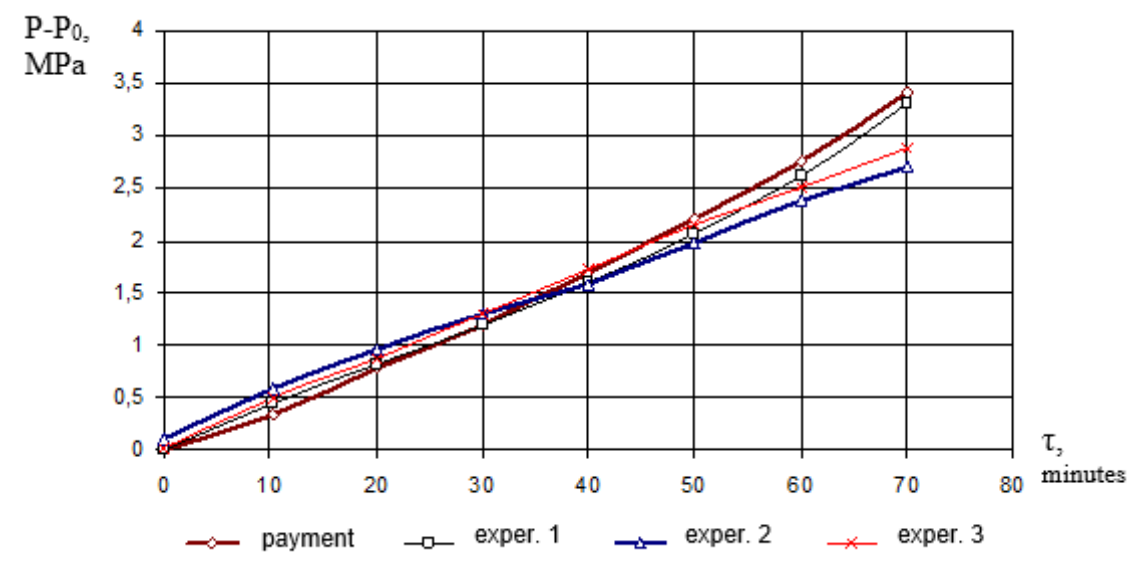

Fig. 4. Dependence of methane pressure in a cryogenic tank on the time of drainage-free storage experiment $1,2,3$

The rate of change of temperature fields over time in the liquid phase of natural gas in a cryogenic tank is shown in Figure 5. 


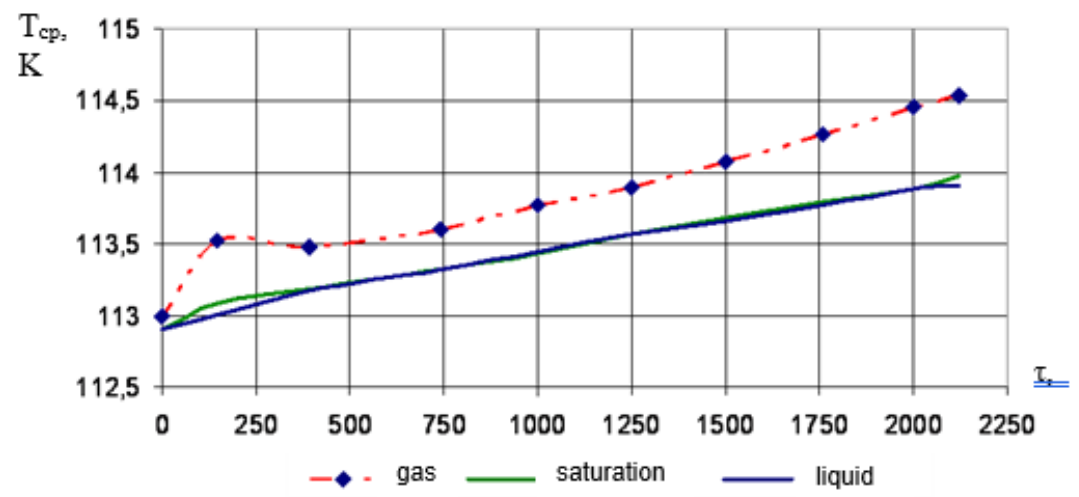

Fig. 5. The dependence of the change in the average temperature (Tcr) of the gas in the saturated and liquid state of the phase over time $(\tau)$.

\section{Discussion}

The results of the calculations and experiments carried out confirmed the convergence with the use of the developed mathematical model, which made it possible to construct the dependences of the change of the main parameters $(\mathrm{P}, \mathrm{T})$ in the cryogenic tank on time. With the use of modern insulation materials and a new method of temperature dumping by the method of cascade throttling, the shelf life of liquefied methane has been increased by more than 1.5-2 times.

\section{Conclusions}

The results of mathematical modeling of the implementation of a new method of regulating the temperature mode of gas in a tube using the cascade throttling method ensured a decrease in gas temperature when discharged into the tube by $1.5 \ldots 3^{\circ} \mathrm{C}$. In this case, the maximum shelf life of liquefied methane in a cryogenic tank using the cascade throttling method was increased to 17 ... 21 days.

\section{References}

1. J.M. Austin, Reaction zones in highly unstable detonations. Proc.Combust.Inst. - vol. 30. - pp. 1849-1857. (2005)

2. V.S. Babkin, A.A. Korzhavin, \& V.A. Bunev, Propagation of premixed gaseous explosion flames in porous media./ Combust. Flame 87. pp. 182-190. (1991)

3. A.I. Belyaev, A.S. Afanasyev, Efficiency of vehicle operation / International journal of economics and financial issues, No 2, pp. 24 - 30. (2016)

4. G. Dixon-Lewis Some observations on the th combustion of methane in premixed flames. 11 (International) on Combustion, The Combustion Institute, Pittsburgh. -pp. 951-958. (1967)

5. A.Yu. Izmailov, O.N. Didmanidze, G.Ye. Mityagin, A.M. Karev Resource saving in road transport. Moscow, (2016) 
6. W. Lee, Doo-Sung Baik, T. Rogers and P. Petersen, Study on Performance and Exhaust Gas Characteristics of Directly Injected CNG Engine. International Journal of BioScience and Bio-Technology Vol.6, No.2, p.179-186. (2014)

7. U. Frost, Heat transfer at low temperatures. Edited by N.A. Anfimov. Moscow: ed. Peace. p. 391. (1977)

8. C.W. Hirt, B.D. Nicholls. Volume of Fluid (VOF) Method for Dynamicsof Free Boundaries // J. Comput. Phys. Vol. 39. p. 201. (1981)

9. R.I. Nigmatulin, Fundamentals of mechanics of heterogeneous media. - Moscow: Nauka, (1978)

10. S. Patankar, Numerical methods for solving problems of heat transfer and fluid dynamics. - Moscow: Energoatomizdat, (1984)

11. R.N. Safiullin, A.S. Afanasyev, Integrated assessment of methods for calculating harm caused by vehicles in transport of heavy cargoes. IOP Conference Series: Earth and Environmental Science. 194(7), 072011, 2018, C.1-5

12. O.M. Belotserkovsky, Yu.M. Davydov. The method of large particles in gas dynamics. Moscow: Nauka, (1982).

13. C. Prakash, S.V. Patankar, A control volume-based finite-element method for solving the Navie-Stokes equations using equal-order velocity-pressure interpolation // Numerical Heat Transfer.Vol. 8. p. 259. (1985)

14. L. Kagan, Effects of hydraulic resistance and heat losses on deflagration-to-detonation transition. In Deflagrative and detonative combustion (eds G.D. Roy \& S.M. Frolov). pp. 157 - 168. (2010)

15. R.T. Khakimov, S.N. Shirokov, M.A. Yefremova, M.V. Kiselev, Innovation approach to ecological problems solution on north-east region. Bulletin of International agricultural education Academy, (24): 83-86. (2015)

16. R.T. Khakimov, O.N. Didmanidze, A.S. Afanasyev, Research of heat generation indicators of gas engines. Electromechanics and Mechanical Engineering / Journal of Mining Institute. - Vol. 229. - P. 50-55. (2018)

17. V. Yakhot, Renormalization group analysis of turbulence: 1 . Basic theory. J. Scientific Computing. - Vol. 1. - No. 1. - P. 1. (1986)

18. X.J. Gu, Laminar burning velocity and Markstein lengths of methane-airmixtures. Combust. Flame1. - Vol. 21. - pp. 41-58. (2000)

19. G.E. Groff, The Cellular Nature of Confined Spherical Propane-Air Flames. Combustion and Flame. - Vo1. 48. - pp. 51-62 (1982)

20. M. Metghalchi, Laminar burning velocity of propane-air mixtures at high temperature and pressure. Combustion and Flame. - vol. 38. - pp. 143-154. (1980)

21. M.I. Hassan, Properties of laminar premixed $\mathrm{CO} / \mathrm{H} 2$ air flames at various pressures. Journal of Propulsion. -. Power 13 (2). - pp. 239-245 (1997)

22. J. Wu, Study Related to Downsize of Production Engine. PhD Thesis. University of Leeds. - pp. 341-348. (2003)

23. C. Serrano, Laminar burning behaviour of biomass gasification-derived producer gas. International Journal of Hydrogen Energy. - vol. 33. - pp. 851-862. (2008)

24. S.Y. Liao, Determination of laminar burning velocities for natural gas. Fuel. - vol. 83(9). - pp. 1247-1250. (2004) 
25. E.A. Mikaelian, Survey of the Gas-Turbine Gas-Compressor Units of Compressor Plants Based on the Modified Thermodynamics Model. International Journal of Alternative Fuels. - vol. 17. - pp. 1125-1131. (2015)

26. S.K. Mahla, Effect of EGR on Performance and Emission Characteristics of Natural Gas Fueled Diesel Engine. Jordan Journal of Mechanical and Industrial Engineering. - vol. 4. - pp. 523-530. (2010) 